\title{
Tiefkalte Helium-Moleküle
}

Wie alle Edelgase hat Helium eine vollständig gefüllte Valenzelektronenschale und kann entsprechend keine kovalente Bindung eingehen. Unter extrem kalten Bedingungen genügt jedoch die äußerst schwache Van-der-Waals-Wechselwirkung um mehrere Heliumatome aneinander zu binden. Einer Gruppe von Atomphysikern der Goethe Universität Frankfurt ist es gelungen, diese Verbindungen herzustellen und zu vermessen.

Die Van-der-Waals-Wechselwirkung ist allgegenwärtig in der Natur. Sie bindet Moleküle und Atome aneinander oder an Oberflächen, ohne dass dafür Elektronen ausgetauscht werden müssen. Ihre Stärke ist abhängig von der Polarisierbarkeit der Bindungspartner, also davon wie leicht die Elektronen gegenüber den Kernen verschoben werden können. Um eine Bindung zu erzeugen genügt bereits eine temporäre Asymmetrie der Ladungsverteilung eines ansonsten nach außen hin neutralen Stoffes.

Auch Helium besitzt, trotz seines kugelsymmetrischen s-Orbitals, zu jedem Zeitpunkt ein temporäres Dipolmoment. Dieses Dipolmoment ist allerdings äußerst gering. Ob die anziehende Kraft, die dieses Dipolmoment auf ein benachbartes Heliumatom bewirkt, ausreicht, um die Nullpunktsenergie (die quantenmechanische Mindestenergie einer Bindung) zu überwiegen, war lange Zeit unklar. Erst 50 Jahre nach der ersten Vermutung seiner Existenz im Jahre 1928 lieferten theoretische Modelle übereinstimmende Ergebnisse, die zeigten, dass ein Helium-Molekül bestehend aus zwei HeliumAtomen in unserem Universum vorkommen sollte. Noch länger dauerte der experimentelle Nachweis: Ein System zu detektieren, das bei der geringsten Interaktion mit seiner Umgebung zerfällt ist schwierig. Die Bindungsenergie des Helium-Moleküls ist milliarden-fach geringer als die alltäglicher Verbindungen wie N2 oder O2. Um die fragilen Helium-Moleküle herzustellen, müssen Heliumatome zu sanften Kollisionen gebracht werden, in denen sie aneinander haften bleiben, ohne dass die gerade erzeugte Bindung direkt wieder zerreißt. Dies kann nur bei einer Umgebungstemperatur nahe des absoluten Nullpunktes geschehen. Zudem muss zunächst der Druck ausreichend hoch sein, da dies die Zahl der Kollisionen und damit die Wahrscheinlichkeit der Erzeugung der Moleküle erhöht. Hingegen sollte - nach erfolgter Bindung - der Druck idealerweise stark abfallen, um weitere Kollisionen zu verhindern, die die gerade erzeugte Verbindung wieder zerstören könnten. Diese ungewöhnlichen Verhältnisse können während der Expansion von Heliumgas durch eine mikrometergroße Düse in ein Vakuum geschaffen werden. 


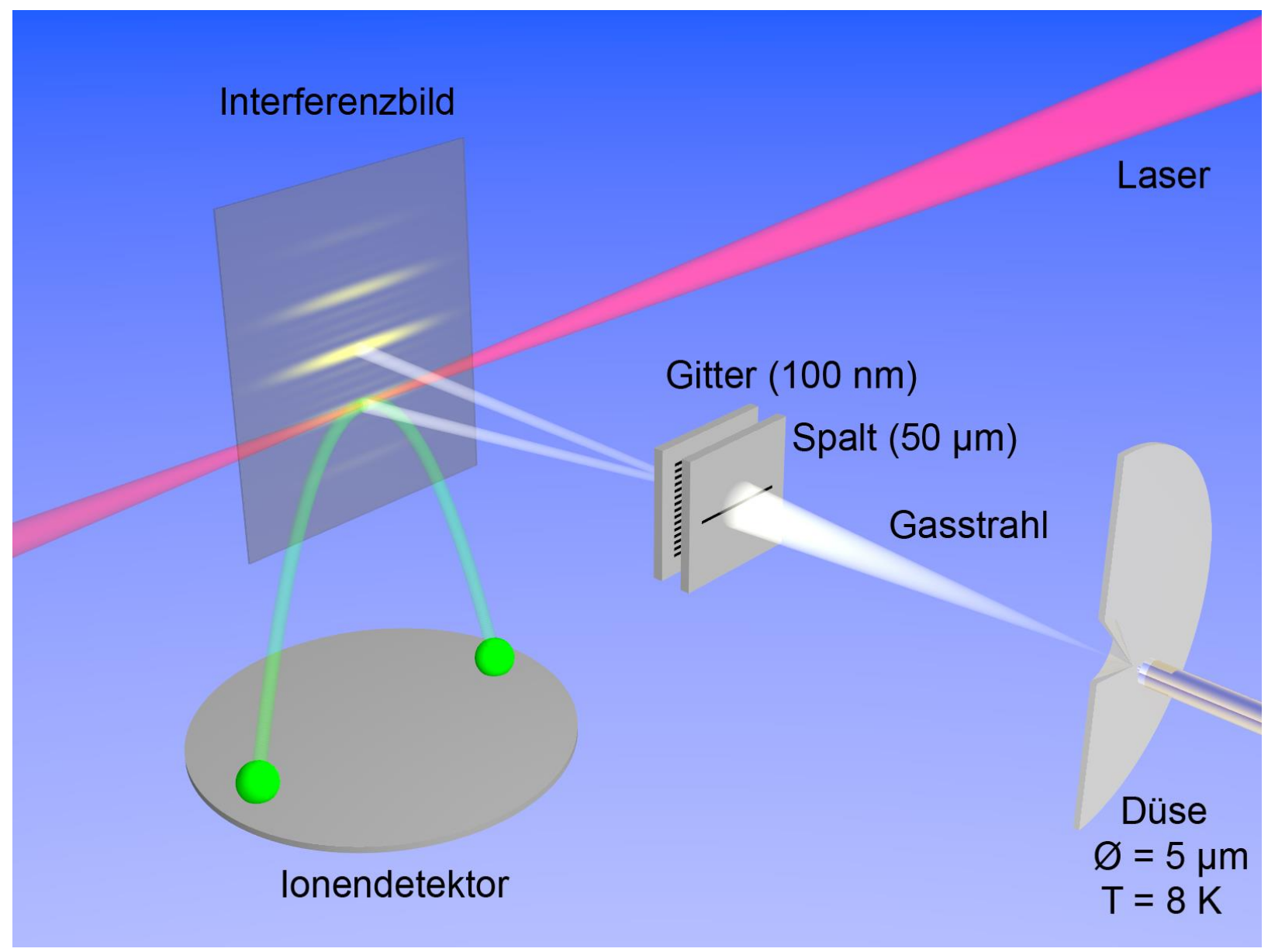

Abb. 1: Schematische Darstellung der Messtechnik mit der die Helium-Moleküle sichtbar gemacht wurden. Die Helium-Moleküle werden in einer Düse bei Temperaturen nahe dem absoluten Nullpunkt erzeugt und anschließend durch ein Beugungsgitter so abgelenkt, dass sie in den Fokus eines Lasers gelangen, wo sie ionisiert werden. Die geladenen Teilchen stoßen sich voneinander ab, und werden von einem orts- und zeitauflösenden Detektor nachgewiesen.

Der Gasstrahl der sich aus einer solchen Düse formt enthält allerdings nicht nur Helium-Moleküle aus zwei Atomen, sondern auch größere Moleküle und vor allem viel ungebundenes, atomares Helium. Aus einer solchen Mischung wurden $\mathrm{He}_{2}$-Moleküle mit Hilfe eines quantenmechanischen Tricks herausgefiltert: Entsprechend dem Welle-Teilchen-Dualismus besitzen auch Atome und Moleküle eine wohldefinierte Wellenlänge. Passieren sie ein Beugungsgitter so entsteht (ganz analog zum klassischen Doppelspaltversuch mit Licht) ein Interferenzbild (siehe Abb. 1). Die Wellenlänge der Teilchen ist hierbei allerdings massenabhängig, so dass sich der gemischte Gasstrahl in mehrere Teilstrahlen unterschiedlich großer Helium-Moleküle auffächert. Dies ist z.B. ähnlich zur Zerlegung von Licht in seine einzelnen Farben durch ein Prisma. Mit dieser Methode konnte die Existenz der $\mathrm{He}_{2}$-Moleküle in den 90er Jahren erstmals experimentell bestätigt werden [1]. Es konnte damals sogar die mittlere Ausdehnung von $\mathrm{He}_{2}$ bestimmt werden, indem winzige Abweichungen in der Auffächerung durch die Interferenz analysiert wurden [2,3]. Anders als bei alltäglichen Molekülen ist die mittlere Bindungslänge von $\mathrm{He}_{2}$ aber nur bedingt aussagekräftig: durch die winzige Bindungsenergie kommen (den Vorhersagen nach) im Grundzustand des Moleküls Bindungsabstände von einigen $\AA$ bis zu einigen hundert $\AA$ vor. Um die 
Verteilung dieser Abstände zu vermessen bedienten sich Physiker der Universität Frankfurt der sog. Cold Target Recoil Ion Momentum Spectroscopy (COLTRIMS) Messmethode. Mit dieser Technik kann der momentane Bindungsabstand eines einzelnen Moleküls bestimmt werden. Hierzu wurden einzelne Moleküle mit einem Laserpuls beschossen, der so intensiv war, dass beide Bindungspartner gleichzeitig ionisiert wurden. Die erzeugten lonen stoßen sich voneinander ab, wobei die Stärke der Abstoßung vom momentanen Abstand der beiden Atome des Moleküls abhängt. Die verwendete COLTRIMSMesstechnik erlaubt es nun, beide Ionen nachzuweisen und deren Energie zu bestimmen. Da die gemessene Energie von der Stärke der Abstoßung abhängt, kann auf den ursprünglichen Abstand zurückgeschlossen werden. Die Abstoßung der Teilchen bewirkt also, dass sich das einzelne Molekül extrem aufbläht und so in der makroskopischen Welt nachweisbar wird. Um die Verteilung der im $\mathrm{He}_{2}$ vorkommenden Abstände zu erhalten, wurde diese Messung mit Millionen einzelner Moleküle wiederholt. Die Verteilung zeigt Erstaunliches: Die Bindungspartner können tatsächlich Abstände von bis zu $250 \AA ̊$ A einnehmen. Das ist so groß, dass ein DNS-Strang oder z.B. kleine Circoviren zwischen den beiden Helium-Atomen hindurch gefädelt werden könnten (siehe Abb. 2). Der riesige Raum zwischen den Atomen ist leer.

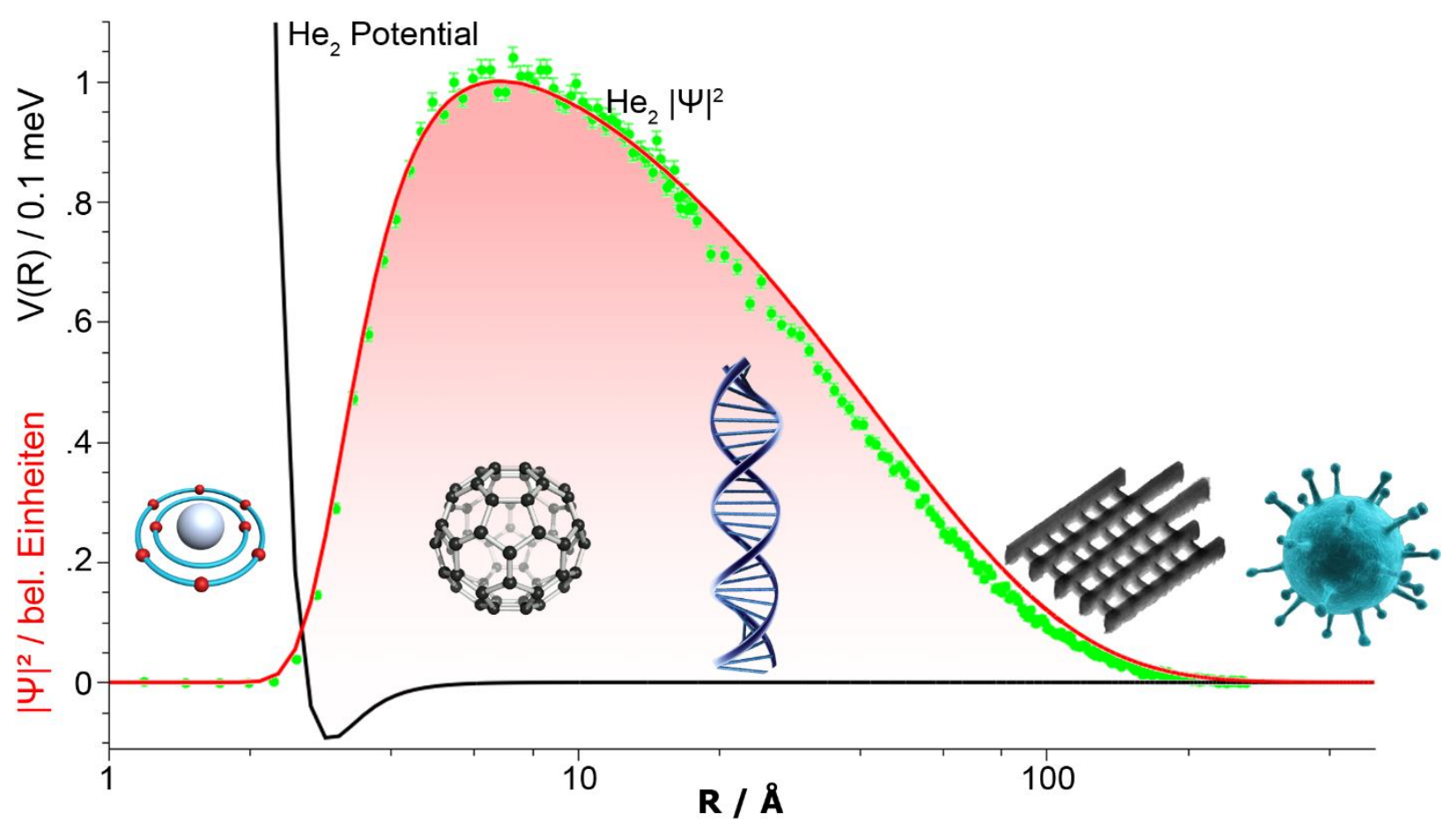

Abb. 2: Gemessene Abstandsverteilung der beiden Atome im He2-Molekül in logarithmischer Darstellung (grün). Abstände von bis zu $250 \AA ̊$ konnten gemessen werden. Im Vergleich eine Modellrechnung (rot) und das He-He Potential (schwarz) [4]. Als Größenvergleich sind dargestellt (von links nach rechts): Sauerstoff-Atom, $\mathrm{C}_{60}$-Fulleren, Durchmesser eines DNS-Strangs, $11 \mathrm{~nm}$ Transistorgeometrie, Circovirus.

Des Weiteren konnte die Vermessung des Helium-Moleküls zeigen, dass die Gesetze der Quantenmechanik genau so wirken, wie sie in theoretischen Modellen beschrieben werden. Sie zeigte z.B., dass in diesem extremen Molekül Effekte eine Rolle spielen, die man ansonsten in der Molekülchemie getrost vernachlässigen kann. Ein solcher Effekt ist etwa Zeitverzögerung der 
Wechselwirkung zwischen Teilchen, die sich aus der Relativitätstheorie ergibt. Die Bilder des Helium Moleküls zeigten außerdem, dass das Molekül nur zu $20 \%$ in einem Bereich existiert, der durch die klassische Physik erklärbar ist. In den restlichen 80 \% der Fälle befinden sich die Bindungspartner bei riesigen Abständen, die ausschließlich durch die Gesetze der Quantenmechanik beschrieben werden können - im sog. "Tunnelbereich".

\section{Literatur:}

[1] Luo F, McBane GC, Kim G, Giese FC, Gentry WR (1993) The weakest bond: Experimental observation of helium dimer. J Chem Phys 98(4):3564-3567.

[2] Luo F, Giese CF, Gentry WR (1996) Direct measurement of the size of the helium dimer. J Chem Phys 104(3):1151-1154.

[3] Grisenti RE, et al. (2000) Determination of the bond length and binding energy of the helium dimer by diffraction from a transmission grating. Phys Rev Lett 85(11): 2284-2287.

[4] Przybytek M, et al. (2010) Relativistic and quantum electrodynamics effects in the helium pair potential. Phys Rev Lett 104(18):183003.

Stefan Zeller, Maksim Kunitski, Jörg Voigtsberger, Till Jahnke, Reinhard Dörner, Univ. Frankfurt

Korrespondenzadresse: Prof. Dr. R. Dörner;

Institut für Kernphysik,

Goethe-Universität Frankfurt am Main;

E-Mail: doerner@atom.uni-frankfurt.de 